\title{
Pertussis outbreak in southern Ethiopia: challenges of detection, management, and response
}

Aychiluhim D. Mitiku', Mesele D. Argaw ${ }^{1 *}$ D, Binyam F. Desta ${ }^{1}$, Zergu T. Tsegaye ${ }^{1}$, Afework A. Atsa $^{2}$, Bekele B. Tefera ${ }^{3}$, Ephrem Teferi ${ }^{1}$, Deirdre Rogers ${ }^{4}$, Ismael A. Beshir ${ }^{1}$, Asrat G. Alemu', Desta A. Ayesa', Derebe T. Abate', Agegnehu G. Sendeku' and Rudzani Muloiwa ${ }^{5}$

\begin{abstract}
Background: Despite the availability of effective vaccines, pertussis remains endemic with high fatality rates in low and middle-income countries (LMIC). This study aims to describe an outbreak of pertussis in a health district of Ethiopia. The study highlights the challenges faced by the health system in identifying pertussis cases and appropriately responding to the outbreak at the district level.

Methods: A descriptive cross-sectional study was conducted using data sourced from the District Public Health Emergency and Management (PHEM) surveillance service and outbreak management field reports. Stratified attack rates and fatality rates for pertussis are described. Systemic problems leading to the outbreak are explored and narrated. A modified CDC pertussis case definition was employed with a polymerase chain reaction used to confirm cases.

Results: From September 2018 to January 2019, 1840 suspected, probable, and confirmed pertussis cases and six deaths were identified. Pertussis cases ranged from 1 month to 51 years in age. An outbreak occurred in 14 out of the 24 villages of Dara Malo district. The overall attack rate was 1708 per 100,000 population with a fatality rate of 3.3 per 1000 pertussis cases. The highest attack rate of 12,689/100,000 was seen in infants. Among confirmed, probable and suspected pertussis cases, only $41.1 \%$ had completed the three-dose pertussis vaccine's primary schedule. The household survey revealed a population coverage of 73.4 and $40.8 \%$ for Pentavalent vaccine dose one and three respectively. Investigations suggested the existence of a poor cold chain management system in the study area.
\end{abstract}

Conclusions: There is an urgent need to build capacity to strengthen routine vaccination services and improve the maintenance of the vaccine cold chain. Other LMICs are urged to take lessons learned from this outbreak to strengthen their own vaccination programs and capacitate health workers to manage local outbreaks.

Keywords: Pertussis, Outbreak, Investigation, Dara Malo, Southern Ethiopia

\footnotetext{
* Correspondence: mdamte5@gmail.com

'USAID Transform: Primary Health Care project, JSI Training \& Research Institute, Inc. in Ethiopia, P.O. Box 1392, code 1110 Addis Ababa, Ethiopia Full list of author information is available at the end of the article
}

(c) The Author(s). 2020 Open Access This article is licensed under a Creative Commons Attribution 4.0 International License, which permits use, sharing, adaptation, distribution and reproduction in any medium or format, as long as you give appropriate credit to the original author(s) and the source, provide a link to the Creative Commons licence, and indicate if changes were made. The images or other third party material in this article are included in the article's Creative Commons. licence, unless indicated otherwise in a credit line to the material. If material is not included in the article's Creative Commons licence and your intended use is not permitted by statutory regulation or exceeds the permitted use, you will need to obtain permission directly from the copyright holder. To view a copy of this licence, visit http://creativecommons.org/licenses/by/4.0/ The Creative Commons Public Domain Dedication waiver (http://creativecommons.org/publicdomain/zero/1.0/) applies to the data made available in this article, unless otherwise stated in a credit line to the data. 


\section{Background}

Pertussis is an extremely infectious vaccine-preventable disease caused by the gram-negative coccobacillus, Bordetella pertussis, and less commonly by Bordetella parapertussis [1]. Pertussis remains endemic and has reemerged as a public health problem in many countries despite decades of high vaccination coverage in infants [2]. Pertussis is widely distributed in many countries throughout the world. Globally, around 24.1 million pertussis cases and 160,700 deaths from pertussis were reported in children younger than five years in 2014. The African region contributed the largest proportion with 7.8 million (33\%) cases and 92,500 (58\%) deaths. Infants and young children have remained most susceptible to pertussis-related morbidity and mortality. Around 5.1 million (21\%) estimated pertussis cases and 85,900 (53\%) estimated deaths were in infants younger than one year $[3,4]$.

In children, pertussis presents classically with paroxysms of cough ending with the characteristic whoop and post-tussive vomiting. However, in young infants, pertussis can initially present as apneic or cyanotic episodes prior to the development of cough [5]. The mode of transmission is person-to-person via aerosolized respiratory droplets or by direct contact with respiratory secretions. In its early catarrhal stage, pertussis is highly contagious, with a secondary attack rate of up to $90 \%$ among non-immune household contacts. Untreated patients may transmit infection for up to three weeks or more following the onset of typical coughing attacks, although communicability diminishes rapidly after the catarrhal stage [6].

The most common complication and the cause of most pertussis-related death is secondary bacterial pneumonia. Young infants are at the highest risk of acquiring pertussis-associated complications [7]. Adolescents and adults are an important reservoir for $B$. pertussis and are often the source of infection for children $[8,9]$.

Vaccination is the best way to prevent pertussis in all age groups [10]. Evidence suggests that high coverage with highly efficacious vaccines leads to high levels of protection in children under five years of age. In contrast, even minor reductions in overall coverage can lead to an increase in cases [11]. Completion of a three-dose pertussis-containing vaccine schedule prevents $80 \%$ of cases and $95 \%$ of deaths. Incomplete immunization has been shown to prevent severe morbidity with one dose and two doses decreasing mortality by 50 and $80 \%$, respectively [12, 13].

In Ethiopia, a pertussis whole-cell vaccine (wP) has been in use since 1980 [14]. From 2007 this has been in a pentavalent formulation that in addition to pertussis whole-cell vaccine, also contains Diphtheria, Tetanus, Hepatitis B, and Haemophilus influenza type B (DTP-
HepB1-Hib1) vaccines. The pentavalent vaccine is given in three doses scheduled at 6, 10, and 14 weeks of birth [14, 15].

Based on World Health Organization (WHO) estimates, the Ethiopian national coverage of one dose of pertussis-containing vaccines in 2018 was 85\%, while that of three doses was $72 \%$, which is less than the average coverage for Sub Saharan countries [10]. This immunization coverage differs greatly between districts and regions of Ethiopia with coverage of 3 doses of pentavalent vaccine ranging from $20.1 \%$ in Afar to $81.4 \%$ in Tigray. The difference in vaccine coverage is partly due to geographical inaccessibility and other social determinants [16]. However, according to the routine health management information system (RHMIS) report, the Dara Malo district health officials reported a higher vaccine coverage with the third pentavalent dose coverage of $103.0 \%$ in $2016 / 2017$ and $94.8 \%$ in $2017 / 2018$. The pentavalent vaccine dropout rates were 3.0 and $3.4 \%$ respectively over the same period [17].

Diagnosis of pertussis, which is largely based on the clinical picture, may be modified by age, history of previous immunization or infection, antibiotic exposure, and concurrent infection with other pathogens [18]. According to the WHO vaccine-preventable diseases 2019 global summary report, there were no reported pertussis cases from 2000 to 2017 in Ethiopia. In 2018, however, there were 2423 cases of pertussis reported in Ethiopia [15]. WHO data is further corroborated by independent data sources that indicated the occurrence of cases of pertussis in Ethiopia [19, 20].

Uncoordinated and poor epidemic response mechanism is a challenge in containing epidemics and in reducing the resultant morbidity, mortality, and economic loss in different countries. This study aims to describe an outbreak of pertussis in the Dara Malo district of Gamo Administrative Zone, Southern Ethiopia, and to investigate possible factors that may have led to the outbreak. In addition, the study explores and highlights the challenges faced by a low and middle-income country's (LMIC) health care system in case identification and management in response to a pertussis outbreak.

\section{Methods}

\section{Study area and population}

Dara Malo is one of 160 districts administered under Southern Nations, Nationalities and Peoples Region (SNNPR), Ethiopia. The district has 24 administrative structures consisting of one urban and 23 rural villages [17]. Based on the 2007 Ethiopian national census, the projected population of Dara Malo district in 2018 was 107,715 with 16,814 under five and 3436 under one year of age [17]. 


\section{Study design and sampling methods}

To investigate the outbreak a cross-sectional descriptive study design was employed. In addition, based on WHO recommendations for evaluation of vaccination coverage a multistage cluster random sampling method was used [21].

All surveillance records collected from 1st September 2018 to 9th January 2019 were reviewed to identify all documented suspected, probable, and confirmed pertussis cases during the period. Health records were reviewed for each of these cases.

The sample size $(n)$ for household survey was calculated using single population formula:

$$
n=\frac{Z^{2} \alpha / 2^{p(1-p)}}{d^{2}} \text { was employed [22]. }
$$

Where $n i$ is sample size, $P$ is the proportion of third dose pentavalent vaccination coverage [23], and $d$ is the margin of error.

The following assumption was used: since $\mathrm{P}$ is $0.53 \%$ $(p=0.53, \mathrm{q}=0.47)$, allowing $5 \%$ for expected margin of error $(d)$ with $95 \%$ confidence level $\left(Z_{\alpha / 2}=1.96\right)$, considering a design effect of 1.5 and $10 \%$ for non-response rate the required sample size $n$ is 631. After allocating the required sample size for each village using probability populational to size sampling method, primary data were collected from households selected using a systematic random sampling technique.

The primary sampling units were Women Development Army (WDA) members or Health Development Army (HAD) members. The WDA strategy was established in 2018 to enhance community health service quality and equity in Ethiopia. WDA is a network of one to five households represented by their team leaders. Based on geographic location of neighbourhoods, each WDA network is comprised of 25 to 30 households [24]. A list of 720 development teams was organized by the Daro Malo District Health Office [17]. For the first stage, 144 development teams were selected using random table numbers. For the second stage, eighteen to twenty four households in six segments (development teams) of villages were selected using a lottery system by local community leaders [25]. Mothers with children aged 523 months of age were interviewed at their homes by trained data collectors.

\section{Data collection}

In the pertussis case review component of the study, data were sourced from the Public Health Emergency and Management (PHEM) database that includes surveillance data on pertussis. Data extracted included information on age, sex, geographical location, immunization status, dates of onset and health facility visits, treatment received, and outcomes of reported pertussis cases. The study reviews available data to explore the cause of the outbreak in terms of a functional cold chain, and vaccines supply systems at the targeted health facilities and district health office.

In addition, to verify the reported immunization coverage, a household survey was conducted. Mothers with children aged 5-23 months of age were interviewed face to face at their homes by trained data collectors. The household survey questionnaires gathered information on sociodemographic characteristics of mothers (caretakers) and the youngest child. Moreover, childhood immunization date and dose related information was obtained from individual health cards as a preferred source. However, in the absence of childhood immunization cards, the mothers' reports (verbal recall) were taken.

The data were collected by four trained health professionals, each with a master's degree in public health $(\mathrm{MPH})$ and prior experience in reviewing documents. In addition, project advisors and program officers from Transform: Primary Health Care (TPHC), a USAID program, oversaw the quality of collected data.

To ensure quality of data collection, training was provided to the data collectors and their supervisors. At the end of every data collection day, a meeting was held between the data collectors and a supervisor to discuss practical problems and issues of major concern. The investigators rechecked the completeness of the collected data on a daily basis.

\section{Case definition}

To confirm pertussis, nasopharyngeal samples were taken from suspected cases by experienced laboratory professionals from the Ethiopian Public Health Institutes and tested using polymerase chain reaction (PCR) for Bordetella pertussis and Bordetella parapertussis (RealStar Bordetella PCR Kit 1.0. Altona Diagnostics). Suspected cases with paroxysmal cough illness for more than 2 weeks were assessed for possible epidemiological links to cases confirmed by a positive PCR for Bordetella pertussis. Pertussis cases reported to the district health office PHEM department were classified by the outbreak investigation team as laboratory-confirmed, probable, or suspected pertussis using modified Center for Diseases Prevention and Control (CDC) criteria [26].

Confirmed: A confirmed pertussis case is defined as acute cough illness of any duration with a positive PCR for B. pertussis, or a case that meets the clinical case definition and is epidemiologically linked directly to a labconfirmed case [23, 24]. In this study, the confirmed pertussis case is an acute cough illness of any duration with a positive PCR for $B$. pertussis.

Probable: A probable case is defined as a cough lasting $\geq 2$ weeks AND paroxysms of coughing, inspiratory "whoop" or post-tussive vomiting AND No laboratory confirmation AND No epidemiologic linkage to a lab- 
confirmed case. In this study, probable pertussis case is a case Cough lasting $\geq 2$ weeks AND Paroxysms of coughing, inspiratory "whoop" or post-tussive vomiting, AND No laboratory confirmation $[10,26]$.

Suspected: A suspected case is defined as a nonimproving cough of 14 days or more or cough of any duration with paroxysms, or cough of any duration with a whoop.

An epidemic of pertussis According to the CDC pertussis epidemic is defined as a situation when two or more cases clustered in time.

- $\quad 22$ PCR confirmed cases clustered in time (within 42 days of each other) and space (e.g. in one building) where transmission is suspected to have occurred in that setting (e.g. nosocomial transmission in a hospital) OR

- 1 PCR confirmed case AND 1 epi-linked case with cough illness lasting $\geq 2$ weeks with one of the following: paroxysms of coughing, inspiratory whoop, or post-tussive vomiting $[10,26]$.

\section{Data management and analysis}

The data were collected in a spreadsheet, cleaned, summarized, and analysed using Microsoft Excel ${ }^{\circledR}$ and Statistical Packaged for Social Sciences Research (SPSS IBM V 20) [27].

Proportions of cases have been presented as percentages. All continuous data were summarized using medians and ranges. The frequency of cases was reported as a case attack per 100,000 population using the 2018 population for Dara Malo as the denominator, while deaths were reported as fatality rates per 1000 cases. Epidemic curves were depicted using the date of onset of paroxysmal cough and date cases were identified by health workers. This analysis shows the propagated nature of the outbreak. Data were reported stratified by age groups and geographical locations and described in terms of time, place, and person.

The household survey data were used to determine population immunization coverage and to verify the accuracy of routine EPI reports. The first and third doses of pentavalent vaccination were recounted from the register, tally sheet, and compared with the caretakers' report. To characterize the report consistency, national recommended categories were used i.e. consistent if the ratio reported was $\geq 90.0$ and $<110.0 \%$; overreported if it was $<90.0 \%$; and underreported if the ratio was $\geq 110.0 \%$ [28].

The result of the quantitative analysis is presented in frequency tables and graphs, while the findings of filed reports and observations were thematically analysed to explain possible factors for the reported pertussis outbreak.

In addition, using ArcGIS 10.8 a spatial analysis was made to show most affected villages using attack rates and case-fatality rates.

\section{Ethical clearance}

Ethical clearance was obtained from both JSI Research \& Training Institute, Inc., and the SNNPR Health Bureau, Ethiopia, Institute Review Boards (IRBs). Permission to conduct the study was obtained from facility managers.

\section{Results}

\section{Rumour investigation}

On 31st August 2018, a community member reported the death of a 72 months old girl with a history of prolonged paroxysmal cough, post-tussive vomiting, and respiratory whoops. Three more children had recently died in the same village, when a similar illness had been observed to affect almost all children in the village during August 2018.

On September 1st, 2018, the Dara Malo district Health Office revitalized the rapid response multi-sectoral committee and reviewed its Risk and Emergency Management Plan. On the same date, the district health office requested technical, and other resources support from Gamo Zone Health Departments, SNNP regional health bureau, Ethiopian Public Health Institute (EPHI), World Health Organization, USAID's Transform and other development partners.

The PHEM core process established and deployed an epidemic investigation team which comprised a field epidemiologist, an integrated disease surveillance response officer, and health extension workers. The team members were familiarized with the case definitions, diagnosis and treatment protocols, strengthening community-based surveillance, and enhancing community mobilization strategies.

\section{Verifying the pertussis outbreak}

The index case, a five-year-old child with unknown vaccination status was identified from Daro Dime village on 1st September 2018. A reported pertussis suspected case presented at Daro Dime Health Center with a complaint of paroxysmal cough, post-tussive vomiting, and respiratory whoop since 2nd August 2018. The source of infection for the index cases was not documented. The case was treated with trimethoprim-sulfamethoxazole (TMPSMZ) with a dose of trimethoprim $8 \mathrm{mg} / \mathrm{kg} /$ day, sulfamethoxazole $40 \mathrm{mg} / \mathrm{kg} /$ day in two divided doses for 14 days. The outbreak investigation team regarded this probable case as the index case of this pertussis outbreak. The district health office and Daro Dime Health Centre instituted an active case search from 4th to 13th September 2018. Over the period, 471 suspected and 
probable pertussis cases were identified in five kebeles. The outbreak investigation team oriented community leaders, health workers, and elders. The active case search was extended through 27th March 2019. Over the whole surveillance period, a total of 1840 suspected, probable, and confirmed pertussis cases including six deaths, were identified.

\section{Descriptive analysis of reported pertussis outbreak}

The pertussis outbreak is described in terms of time, place, and person.

\section{Demographic distribution of the cases}

From 1st September 2018 to 9th January 2019, there were a total of 1840 reported cases of pertussis. Of the reported cases $9(0.5 \%)$ were confirmed (all due to Bordetella pertussis) while $454(24.7 \%)$ were probable cases and the remaining1377(74.8\%), suspected cases. Dara Dime village reported 838 (45.5\%) of the pertussis cases. The sociodemographic characteristics of the study participants are shown in Table 1 . The age of the cases ranged from 1 month to 50 years of age with a median of 36 months. Of the total cases, 230 (12.5\%) were aged less than six months while 801 (42.5\%) were 1 to 4 years old. Table 1.

The overall attack rate was $1708 / 100,000$. The attack rates by age category were $12,689 / 100,000,5965 / 100$, 000 , and $667 / 100,000$ reported in under one-year infants, 1 to 4 years children, and 5 or more years of children and adults, respectively. Additional file 1 .

A total of six deaths due to pertussis were reported to give a total case fatality rate of 3.3 deaths per 1000 cases. These include five deaths from Daro Dime and one death from Menena Aba kebele. Two deaths each were under 6 months, and between 6 to 12 months. One death each was from 1 to 4 years and 5 to 9 years. The highest case fatality rate was found among infants less than 5 months of age with a case fatality rate of $8.70 / 1000$ cases, followed by infants aged 6 to 11 months with 4.85/1000 cases. Figure 1.

\section{Clinical presentation}

The most frequent presentation was paroxysmal cough which was a complaint in all $1840(100.0 \%)$ cases. This was followed by post-tussive vomiting which was reported in $1579(85.8 \%)$ cases. Inspiratory whoop was reported in $454(24.7 \%)$ while $136(7.4 \%)$ had a low-grade fever. In addition, 37(2.0\%) of the cases had additional signs and symptoms indicative of severe illness. The most frequently reported signs of severity were syncope and apnea in eight $(21.6 \%)$ and six (16.2\%) cases, respectively. Figure 2.

\section{Descriptive analysis by time}

Figure 3 presents the Epi-Curve which plots the frequency of pertussis cases over time by date of onset of paroxysmal cough and date cases were identified by health workers, respectively. The epi-curve presented clearly demonstrates the classic propagated or progressive source nature of the pertussis outbreak, where three peaks are observed over time. The mean delay to seek medical care by patients or caretakers was 9.4 days, with a median of 7 days, ranging from one day to 63 days.

\section{Geographical distribution of the outbreak cases}

The highest attack rate was reported from Dara Dime village while the highest case fatality rate was noted in Menena Aba village. Figure 4 depicts ArcGIS 10.8 spatial analysis pertussis case attack rates and case fatality rates by a village.

\section{Vaccination status}

The number of cases who had received a pertussiscontaining vaccine was found to be 169 (9.2\%), 321 (17.4\%), and 761 (41.4\%) for one dose, two doses, and three doses, respectively. Of the rest of the reported pertussis cases, $232(12.6 \%)$ had not received any vaccine dose while 357 (19.4\%) had unknown immunization status. For individuals who received three doses of the pentavalent vaccine, a median of 41 (Interquartile range 19-67) months had elapsed between the last dose and the current illness.

Table 1 Socio-demographic characteristics of Pertussis cases in Dara Malo District, Gamo Zone, SNNPR, Ethiopia, August 2018January 2019

\begin{tabular}{llll}
\hline Variable & Characteristics & $n=1840(\%)$ & Total population \\
\hline Sex & Male & $883(48.0)$ & 54,935 \\
& Female & $957(52.0)$ & 52,780 \\
Age groups & $\leq 5$ months & $230(12.5)$ & 1615 \\
& $6-11$ months & $206(11.2)$ & 1821 \\
& $1-4$ years & $801(43.5)$ & 13,378 \\
$5-9$ years & $457(24.8)$ & 14,603 & 13,388 \\
& $10-14$ years & $136(7.4)$ & 62,910 \\
& $\geq 15$ years & $10(0.5)$ & \\
\hline
\end{tabular}




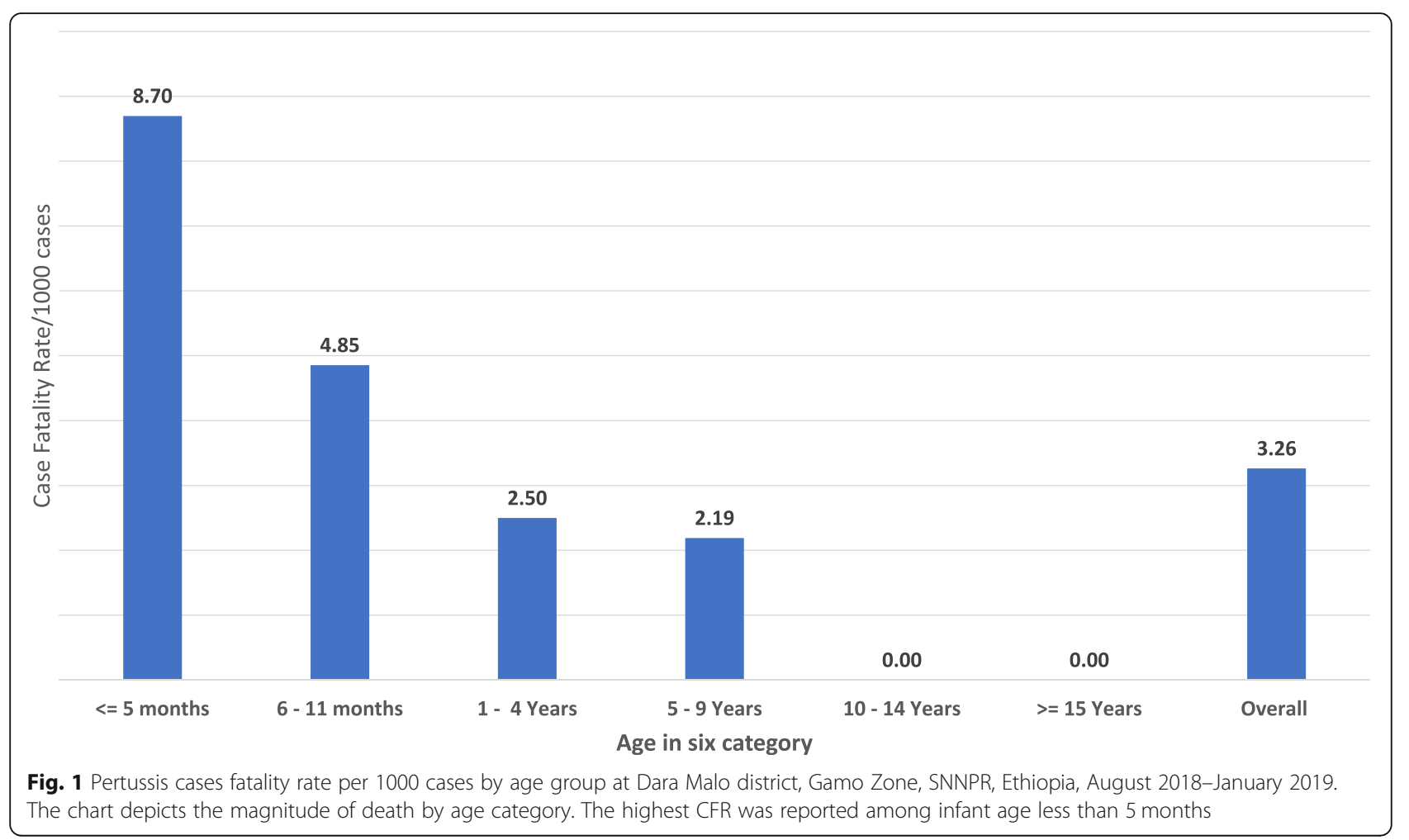

\section{Management of the pertussis outbreak}

There were delays in notification and lack of timely proper management of probable or suspected pertussis cases in the community. As the health workers were treating cases as pneumonia, pertussis was never suspected until the death of four children occurred due to the outbreak.

The outbreak response team lobbied community leaders and elders to mobilize the community to report cough of 2 weeks or more to nearby health

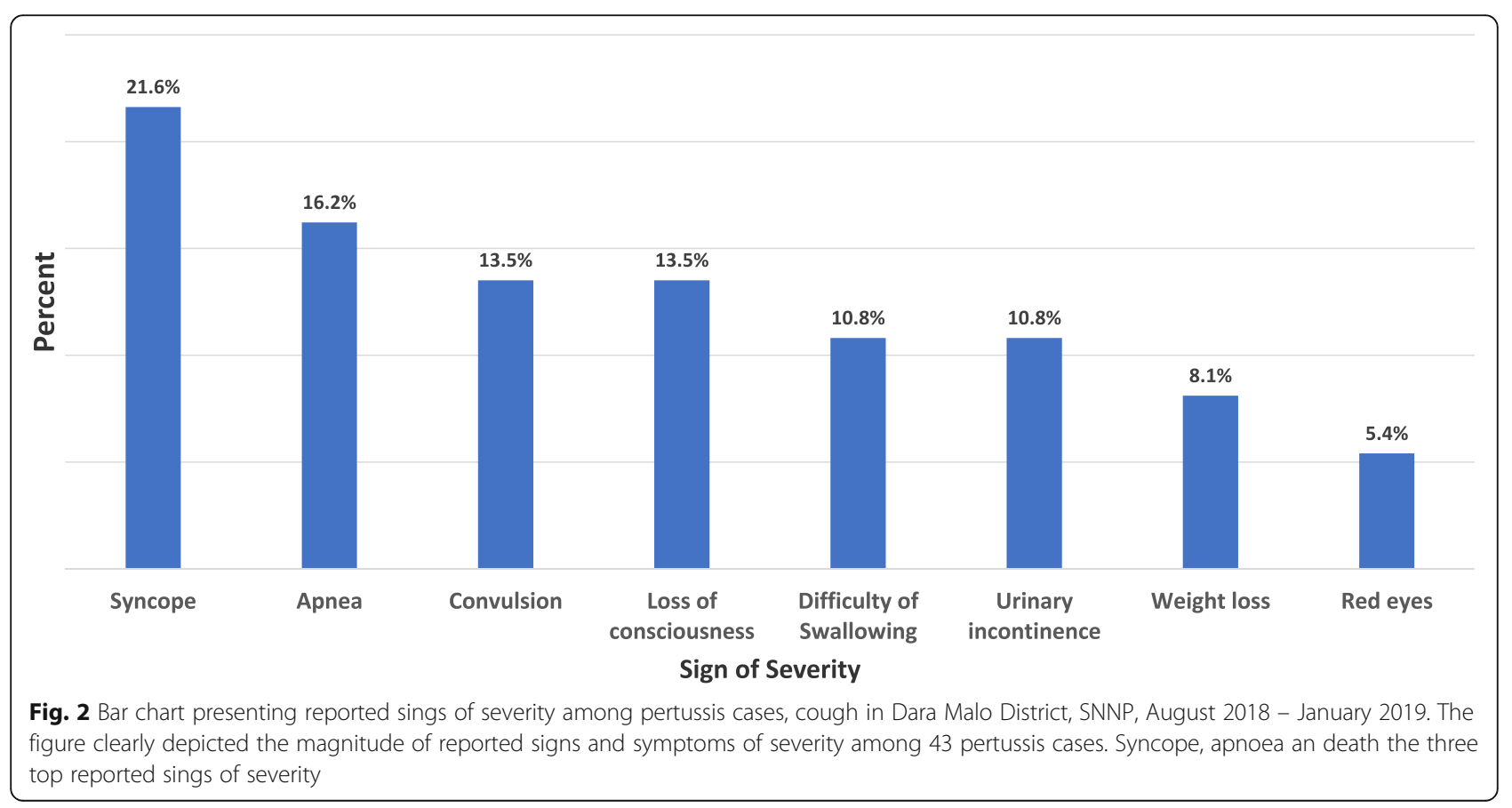




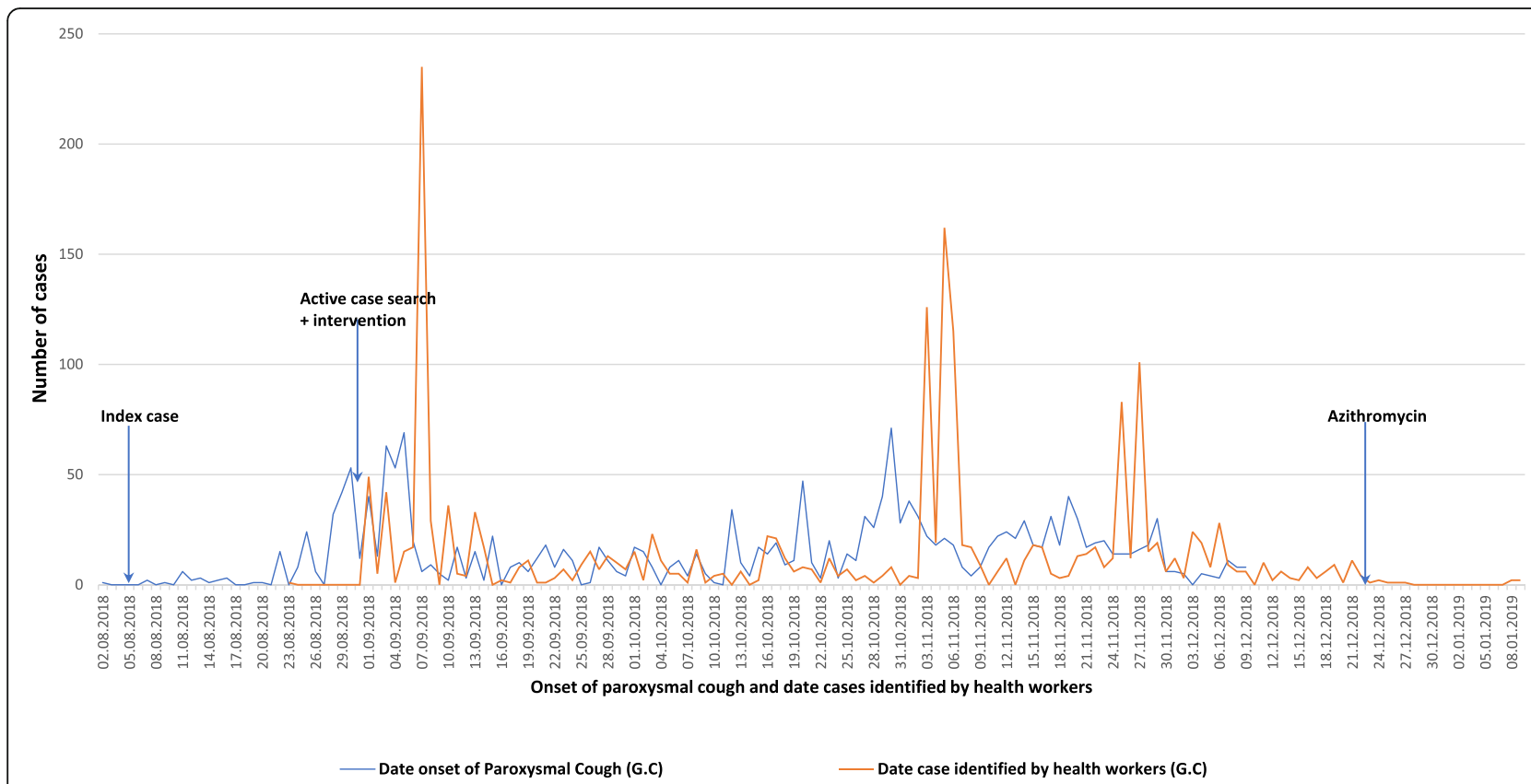

Fig. 3 Epi Curve of Pertussis cases by date of onset of paroxysmal cough and identified by health workers in Dara Malo District, SNNP, August 2018 - January 2019. The epi-cure by date of onset of paroxysmal cough showed three peaks, which shows the propagative nature of the spread of pertussis infection. In addition, the epi-cure by date of pertussis case identified by health workers showed three peaks, which shows the long period taken to control the spread of infection

posts. Information leaflets were developed and distributed to all household members using the local language, Gamo.

Individual cases were treated with antibiotics to reduce the severity and duration of symptoms, and to prevent complications. From 1st September to 22nd December 2018,
1832 (99.5\%) of the identified pertussis cases were treated with amoxicillin, cotrimoxazole, or erythromycin. The outbreak response was hindered by a lack of sufficient erythromycin syrup doses for children under five years of age.

In order to reduce the spread of infection, a Mass Drug Administration (MAD) program was initiated

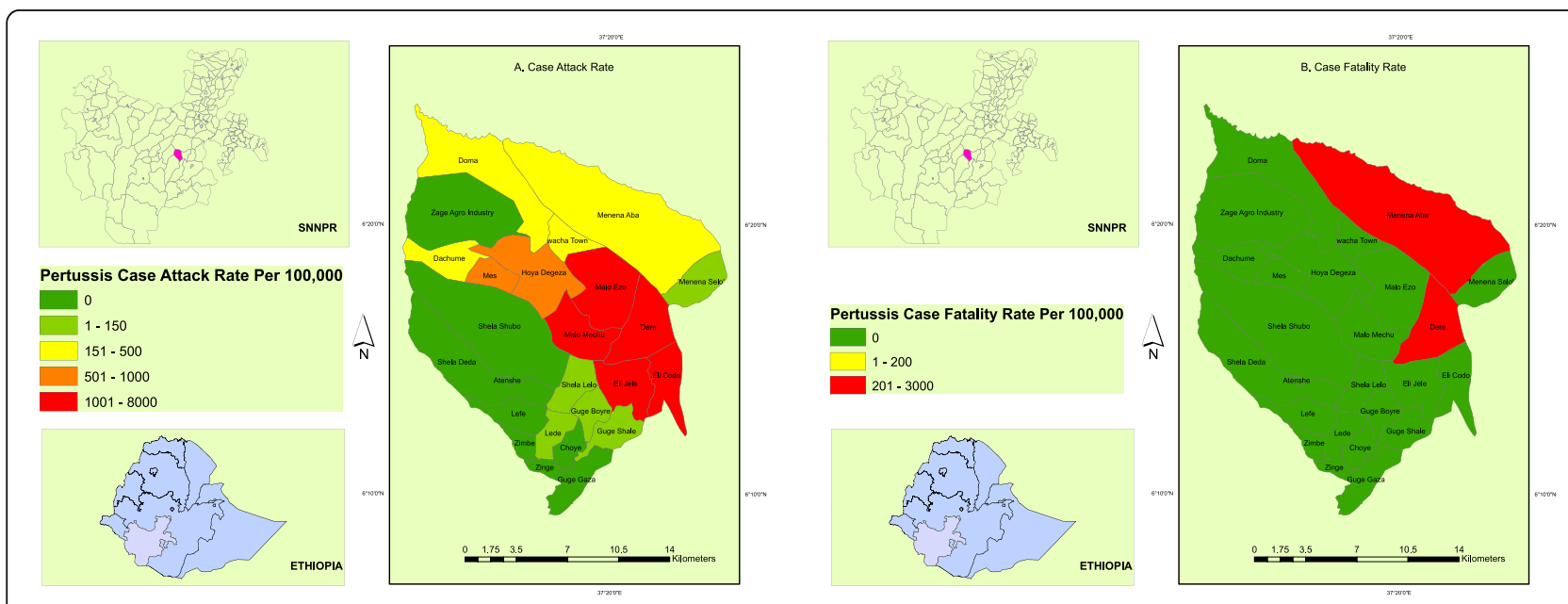

Fig. 4 Pertussis Case Attack Rate (a) and Case Fatality Rate (b) by Village (Kebele), Dara Malo district, Gamo Zone, SNNP region, Ethiopia August 2018-January 2019. The ArcGIS map presents the location of Ethiopia, SNNPR and Dara Malo district. In addition, the Pertussis Case Attack rate and Case Fatality rates were presented with spatial analysis by village (Kebele). The map can be reproduced or used after acknowledging the authors. (Source: Shape file from Ethiopia Central Statistical Agency (CSA), 2016 at https://africaopendata.org/dataset/ethiopia-shapefiles) 
following a facilitated two days training for 110 health workers including 90 drug distributors, $17 \mathrm{su}$ pervisors, and three district coordinators. The MDA was conducted from 23 to 27 December 2018 using oral azithromycin at a daily dose of $10 \mathrm{mg} / \mathrm{kg}$ under the direct supervision of health workers on the first day followed by $5 \mathrm{mg} / \mathrm{kg}$ from 2 nd to 5 th day. This prophylactic mass campaign targeted 69,587 residents of the 14 pertussis-affected villages. Directly observed azithromycin therapy was received by 67 , 236 (96.6\%) people under the supervision of health care providers. Among these, 21,003 (31.2\%) were under 10 years of age. The campaign required $\$ 18$, 325.00 USD of investment from the district health office and partners for its successful implementation.

\section{Prevention of spread of infection}

EPI register reviews and oral responses of patients or caretakers were counted and mapped for supplementary immunization campaigns. After completing prophylactic antibiotic treatment with azithromycin, the outbreak management team organized a pentavalent vaccination campaign from the 24th to the 28th of January 2019. Three supplementary immunization campaigns were organized. Children less than two years were vaccinated, including 1697 who received their first dose, 2564 their second dose, and 3287 their third dose of pertussiscontaining vaccine.

All schools were closed for the period of mass preventive prophylaxis campaigns while community members were advised to avoid participating in local markets. Community members were advised to implement strict personal hygiene including frequent hand washing and avoiding contact with pertussis suspected individuals.

Following the last reported case, the District Health Office continued its active case search for more than two months on a daily basis. The outreach investigation team continued to provide support for individual case management and mass health education for community members. It also continued to enhance the capacity of health workers on cold chain management, defaulter tracing, and use of data for decision making. After no further cases of pertussis were reported from 10th January to 27th March 2019, the investigation team was demobilized.

\section{Formulation of the hypothesis}

The outbreak investigation team reviewed the surveillance reports filled by four health centres, fourteen health posts and six private health facilities before being organized by Dara Malo District Health Office. Based on the routine surveillance report, the completeness rate was assessed as high, with a completeness rate of $84.0 \%$ in $2016 / 17$; $88.0 \%$ in 2017/2018; and $91.0 \%$ in $2018 /$ 2019. Poor housing conditions, reducing vaccine potency, and low vaccination coverage status of affected community were identified as a potential factors contributing to the pertussis outbreak.

\section{Pentavalent vaccination coverage}

Three hundred- and thirty-six facility months' data were extracted. This was supplemented by survey data of 595 children aged 7-23 months, with a response rate of $94.3 \%$. The immunization status on the health cards or caretakers' oral reports on household surveys was compared to that sourced from register tallies. The household survey revealed a 73.4 and $40.8 \%$ vaccination coverage for pentavalent one and three doses, respectively. At the district level, EPI registers for the first and third doses of pentavalent indicated coverages of 95.2 and $104.2 \%$, respectively. Register tallies overreported vaccination coverage by $50.8 \%$ for first pentavalent dose and by $45.1 \%$ for the third dose when compared to health card records or oral reports (Fig. 5).

\section{Environmental factors}

The housing condition and family size per household were assessed. The average house in the affected community had 7 people and lacked air circulation. Community members were encouraged to open ventilate their homes.

\section{Cold chain, immunization services management}

Wacha Health Centre has a high-tension electric power supply. Three out of four health centres have solar panels to obtain uninterrupted electric power for cold chain maintenance. All fourteen health centres collected vaccines for scheduled vaccination campaigns and outreach services. The investigation team did not find continuously recorded temperature monitoring tools. In addition, the thermochromic label on vials or vaccine vial monitor (VVM), revealed that the vaccines were kept at temperatures that do not preserve vaccine potency. Overall, cold chain maintenance was generally found to be poor.

\section{Discussion}

This study confirms a pertussis outbreak that occurred between September 2018 and March 2019, in Dara Malo, a district in Southern Ethiopia. Pertussis outbreak cases occurred in 14 of 24 villages in the district and involved 1840 cases with six deaths. Pertussis is one of the under-reported major causes of morbidity and mortality among children in many low-income countries [3]. This report confirms the importance of strengthening community-based active surveillance programs and the need to enhance the capacity of community health workers in identifying pertussis suspected cases, as well 


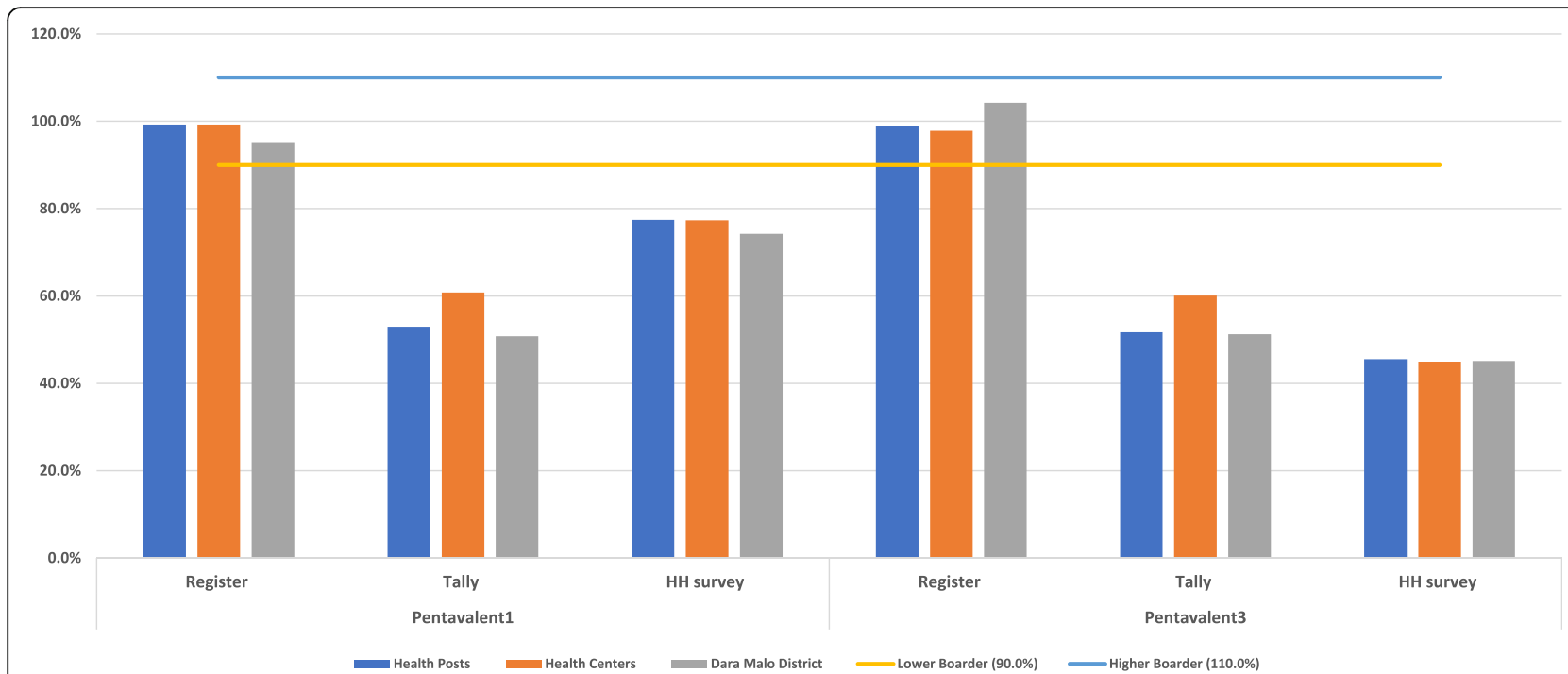

Fig. 5 Verification of pentavalent one and three vaccination services report against household survey, July 2018 - June 2019. The bar chart shows the accuracy of routine EPI report against household survey data at health post, health centre and district level

as providing a prompt effective response to any outbreaks [29, 30].

The current outbreak could have been the result of poor vaccination coverage and a non-functional cold chain management system, combined with a lack of standardized national pertussis responsiveness and management guidelines. Although almost $90 \%$ of cases were older than 5 months, only $41 \%$ of them had received the three doses of a pertussis-containing vaccine as would have been appropriate for age. This report was in line with the population coverage revealed through household survey. In addition, there had been a long interval between the third pertussis vaccine dose and acquiring infection (median of 41 months); hence, the outbreak might have occurred as a result of waning vaccine acquired immunity overtime [31-33].

Though the district health officials confirmed the presence of a high rate of community-based surveillance in weekly reports on public health notifiable diseases over the duration of preceding year, the health systems did not identify the unusual health condition which occurred in Dara Malo district. This finding might have occurred due to the limited capacity of the health care system in identifying risks, developing emergency preparedness plans, as well as diagnosis and treatment of individual cases, prompt response, and management capacity.

The signs and symptoms of both uncomplicated and severe cases were consistent with either CDC or WHO pertussis case definitions for suspected pertussis cases. The cause of this outbreak was confirmed to be due to Bordetella pertussis on PCR. The overall pertussis case attack rate of 1708 per 100,000 inhabitants of Dara Malo district was much higher than the previously documented pertussis attack rate of 130 per 100,000 population reported in South Wollo, North Eastern Ethiopia in 2017 [19] or the 400 per 100,000 population reported in Papua New Guinea in 2012 [34]. The high attack rate documented in this investigation could be due to the case definition, which in addition to confirmed pertussis included both probable and suspected cases. Once laboratory confirmation of the outbreak was made, subsequent cases were clinically diagnosed. This practice is different from other studies, in particular those from high-income countries, in which only laboratoryconfirmed and epidemiologically linked cases are considered. The highly sensitive case definition used would also have led to a relatively low case fatality rate compared to that described for pertussis in other Sub Saharan settings $[35,36]$.

Our report shows that there was a delay in appropriate management to control the outbreak using a nationally recommended and effective antibiotic regimen for individual cases [37]. Contact tracing was largely incomplete and the intervention did not include prophylactic antibiotic administration to susceptible community members until late in the outbreak. Failure to protect close contacts of both confirmed and probable pertussis cases might have encouraged the spread of infection over an extended period. By the time mass prophylaxis started, the outbreak was already dying out naturally. This is indicative of the lack of health system capacity to respond and manage the outbreak timeously. 
The low health-seeking behaviour of the community (as indicated by the more than two months of delay in visiting the health facility recorded in some instances) was most likely due to lack of knowledge on the severity and risk of pertussis, and would have served to exacerbate the situation. As expected, the highest attack rate was in infants followed by children aged one to four years. This finding was consistent with the report of Yeung et al's global estimates [3] and the report of the outbreak in the South Wollo area of Ethiopia [19].

The overall pertussis case fatality rate of 3.3 per 1000 pertussis cases was much lower than the 37 per 1000 pertussis cases from Mekdela [19] and the 30 per 1000 pertussis cases reported from Papua New Guinea (2012) [34]. Four of the six deaths were reported before outbreak control interventions were initiated. The early initiation of the responsive program may have averted further deaths. All cases identified during the outbreak were treated with antibiotics which consisted of amoxicillin, and cotrimoxazole, erythromycin, or azithromycin. The use of antibiotics would have reduced the possibility of severe pneumonia which can occur as a complication of pertussis infection [30].

The cause of this outbreak could be multifaceted. Even though the RHMIS reported a high rate of vaccination coverage of over $95 \%$ with three doses of a pertussiscontaining vaccine in 2016/17 for Dara Malo district., a large proportion of pertussis cases were not fully vaccinated. The inconsistency between routine RHMIS and outbreak data may be due to poor data recording. Maintaining high vaccine coverage is one of the most important interventions in reducing pertussis cases and consequently averting outbreaks [11, 38, 39]. The poor vaccine vial monitoring observed during the outbreak investigation and non-functional cold chain management system at primary health care units may have resulted in poor vaccine potency even in cases that received all doses $[40,41]$.

\section{Limitations}

The study was limited by a lack of resources to confirm the majority of suspected cases which would have affected the diagnostic accuracy of cases; however, in an outbreak, clinical criteria have been shown to have high sensitivity and specificity in the diagnosis of pertussis [42]. We also noted the atypical spikes in the epidemic curve that could be a result of either an artifact created by intermittent augmentation of surveillance practice or real individual clusters within the overall outbreak. In addition, this study did not capture the reason for the delayed health-seeking behaviour of caretakers and the completeness, consistency, and reliability of immunization data. As immunization records were not always available, information on vaccination status could have been affected by recall bias.

\section{Conclusions}

There is an urgent need to build capacity to strengthen routine vaccination services, including defaulter tracing, to keep vaccine coverage at a high level to avert outbreaks. Vaccinating all children with appropriate doses for age must be supplemented with building and strengthening the capacity of health workers to timeously and properly manage outbreaks when these occur. In addition, static and outreach services, community-based surveillance activities, and cold chain maintenance need to be prioritized. A review of data quality status and capacity to respond to outbreaks at the district level must urgently be undertaken. Other LMICs, especially those in Sub-Saharan Africa, should use the lessons from this outbreak to actively review their vaccination systems and promote contextually suitable ways of outbreak detection, diagnosis, management and responses to avert similar challenges [43].

\section{Supplementary information}

Supplementary information accompanies this paper at https://doi.org/10. 1186/s12889-020-09303-2.

Additional file 1: Supplementary file 1. Pertussis cases and attack rates by village with age group in Dara Malo district, Gamo Zone, SNNPR, Ethiopia, August 2018-January 2019.

\section{Abbreviations}

AR: Attack Rate; BCC: Behavioural Change Communication; CDC: Centers for Diseases Control and Prevention; CFR: Case Fatality Rate; EPI: Expanded Programme on Immunization; FMOH: Federal Ministry of Health; HAD: Health Development Army; HEW: Health Extension Workers; HMIS: Health Management Information System; HP: Health Post; IEC: Information Education and Communication; IRB: Institution Review Board; MDA: Mass Drug Administration; MDG: Millennium Development Goal; PCR: Polymerase Chain Reaction; PHCU: Primary Health Care Unit; PHEM: Public Health Emergency and Management; SNNPR: Southern Nations, Nationalities, and Peoples' Region; UNICEF: United Nations Children's Fund; USAID: United States Agency for International Development; VPD: Vaccine Preventable Disease; WDA: Women Development Army; WHO: World Health Organization

\section{Acknowledgements}

USAID Transform: Primary Health Care project is implemented by a consortium of organizations which includes Pathfinder International, JSI Research \& Training Institute, Inc., EnCompass, Malaria Consortium, Abt Associates Inc., and Ethiopian Midwifes Association in collaboration with local Government and Non-government partners. The investigators are indebted to all participants for their cooperation during data collection. We would like to thank Dr. Rebecca Sher and Heran Demissie for language editing the final manuscript.

\section{Authors' contributions}

The authors of this manuscript are ADM, MDA, BFD, ZTT, AAA, BBT, ET, DR, IAB, AGA, DAA, DTA AGS and RM. ADM, MDA, BFD, BBT, DR and RM made a substantial contribution to conceiving and designing the study. ADM, MDA, $A A A, I A B, A G A, D A A$ and $A G S$ was responsible for data curation and overseeing the field work. MDA, DTA, and RM cleaning the data, analysing the data, and interpreting the analysis. The project was administered by BFD, ZTT, ET and AGS. The original draft of this manuscript was developed by 
MDA. All authors read and approved the final manuscript. MDA, the corresponding author, submitted the manuscript for publication.

\section{Authors' information}

ADM: MPH; Expanded Immunization Program Advisor at USAID Transform: Primary Health Care, JSI Research \& Training Institute, Inc., Addis Ababa, Ethiopia.

MDA: Ph.D.; Senior Health Systems Strengthening Advisor at USAID Transform: Primary Health Care, JSI Research \& Training Institute, Inc., Addis Ababa, Ethiopia.

BDF: MPH; Deputy Chief of Party at USAID Transform: Primary Health Care, JSI Research \& Training Institute, Inc., Addis Ababa, Ethiopia.

ZTT: MPH; Programs Director at USAID Transform: Primary Health Care, JSI Research \& Training Institutes, Inc., Addis Ababa, Ethiopia. BBT: PhD, Research Advisor at USAID Transform: Primary Health Care, Pathfinder International, Addis Ababa, Ethiopia.

AAA: BSc Nurse, Public Health Emergency Management Core Process Owner, Dara malo district Health Office, Wacha Town, Ethiopia.

ET: MD, Pediatrician; Child Health Development Advisor at USAID Transform: Primary Health Care, JSI Research \& Training Institute, Inc., Addis Ababa, Ethiopia

DR: PhD, Monitoring and Evaluation Senior Advisor at JSI Research \& Training Institute, Inc., Boston, USA.

IAB: MPH Monitoring and Evaluation Advisor at USAID Transform: Primary Health Care, JSI Research \& Training Institutes, Inc., Addis Ababa, Ethiopia. AGA: MPH; Child Heath Development Officer at USAID Transform: Primary Health Care, JSI Research \& Training Institutes, Inc., Hawassa, Ethiopia. DAA: MPH; Program Officer at USAID Transform: Primary Health Care, JSI Research \& Training Institutes, Inc., Arbaminch, Ethiopia.

DTA: MPH; Monitoring and Evaluation Assistant Director, Abt Associates Inc., Addis Ababa, Ethiopia.

AGS: MPH; Regional Manager at USAID Transform: Primary Health Care, JSI Research \& Training Institutes, Inc., Hawassa, Ethiopia.

RM: MBChB, FCPaeds (SA), MSc. (LSHTM), Groote Schuur Hospital \& University of Cape Town, G5 New Groote Schuur Hospital Building, Anzio Road, Observatory Cape Town, South Africa.

\section{Funding}

This research is a part of the operation research for the United States Agency for International Development (USAID) funded Transform: Primary Health Care project. Under cooperative agreement number of AID-663-A-17-00002. The authors ADM, MDA, BFD, ZTT, BBT, ET, DR, IAB, AGA, DAA, and AGS received salary support from the project. This technical pertussis outbreak investigation report is made possible by the generous support of the American people through USAID. The authors' views expressed in this technical report do not necessarily reflect the views of USAID or the United States Government. The funder does not have any role in the design of this study, data collection, analysis and writing of the manuscript.

\section{Availability of data and materials}

The datasets was collected from the Public Health Emergency and Management (PHEM) database and used and/or analysed during the current study are available from the corresponding author on reasonable request.

\section{Ethics approval and consent to participate}

Ethical clearance was obtained from John Snow Inc. with certificate reference number: IRB \# 19-18 and from the Southern Nations, Nationalities and Peoples Regional Health Bureau with reference number: ZS/1-6/36/22. Permission to conduct the research and access PHEM database was obtained from the local health office and primary health care units. Informed written consent was obtained from all participants. To maintain the confidentiality of collected data, anonymity was maintained throughout the research process.

\section{Consent for publication}

Not applicable.

\section{Competing interests}

The authors declare that they have no competing interests.

\section{Author details}

${ }^{1}$ USAID Transform: Primary Health Care project, JSI Training \& Research Institute, Inc. in Ethiopia, P.O. Box 1392, code 1110 Addis Ababa, Ethiopia. ${ }^{2}$ Daro Malo Woreda Health Office, Dara Malo, Ethiopia. ${ }^{3}$ USAID Transform: Primary Health Care project, Pathfinder International, Addis Ababa, Ethiopia. ${ }^{4}$ JSI Research \& Training Institute, Inc., Boston, USA. ${ }^{5}$ Department of Paediatrics \& Child Health, Groote Schuur Hospital \& University of Cape Town, Cape Town, South Africa.

Received: 14 November 2019 Accepted: 27 July 2020

Published online: 11 August 2020

\section{References}

1. Muloiwa R, Kagina BM, Engel ME, Hussey GD. The burden of pertussis in low-and middle-income countries since the inception of the expanded Programme on immunization (EPI) in 1974: a systematic review protocol. Systematic reviews. 2015;4(1):62.

2. World Health Organization. Pertussis vaccines: WHO position paper - august 2015. Wkly Epidemiol Rec. 2015;90(35):433-60.

3. Yeung KH, Duclos P, Nelson EA, Hutubessy RC. An update of the global burden of pertussis in children younger than 5 years: a modelling study. Lancet Infect Dis. 2017;17(9):974-80.

4. Faulkner A, Skoff TH, Martin SW, Cassiday PK, Tondella ML, Liang JL. Manual for the surveillance of vaccine-preventable diseases. Chapter 10: Pertussis; 2015

5. World Health Organization. Surveillance standards for vaccine-preventable diseases. Geneva: World Health Organization; 2018.

6. World Health Organization. Vaccine-preventable diseases (pertussis) surveillance standards. Pertussis Geneva: World Health Organization; 2018. https://www.who.int/immunization/monitoring_surveillance/burden/vpd/ WHO_SurveillanceVaccinePreventable_16_Pertussis_BW_R2.pdf?ua=1 (accessed on 21 May 2019).

7. Centers for Disease Control and Prevention. Epidemiology and prevention of vaccine-preventable diseases, vol. 2. Washington DC: Public Health Foundation; 2015. p. 20.

8. Wiley KE, Zuo Y, Macartney KK, McIntyre PB. Sources of pertussis infection in young infants: a review of key evidence informing targeting of the cocoon strategy. Vaccine. 2013;31(4):618-25.

9. Roush RW, Murphy TV, Are V. TV, Vaccine-Preventable Disease Table Working Group. Historical comparisons of morbidity and mortality for vaccine-preventable diseases in the United States. InJama 2007.

10. Centers for Disease Control and Prevention. In Prevention: vaccine. USA; 2017. https://www.cdc.gov/pertussis/about/prevention/index.html (accessed on 21 May 2019).

11. Miller E, Siegrist C, Tharmaphornpilas P. WHO SAGE pertussis working group background paper SAGE; 2014.

12. Crowcroft NS, Stein C, Duclos P, Birmingham M. How best to estimate the global burden of pertussis? Lancet Infect Dis. 2003;3(7):413-8.

13. World Health Organization. SAGE pertussis working group background paper. In World Health Organization, Geneva, Switzerland https://www.who. int/immunization/sage/meetings/2014/april/1_Pertussis_background_ FINAL4_web.pdf (accessed on 21 May 2019).

14. Federal Ministry of Health (FMOH). Comprehensive Multiyear Plan 2016 2020. In: Ethiopia National Expanded Programme on immunization. Addis Ababa: Federal Ministry of Health. p. 2015.

15. World Health Organization. Immunization monitoring global summary. Geneva: World Health Organization. http://apps.who.int/immunization_ monitoring/globalsummary/countries?countrycriteria\%5Bcountry\%5D\%5B\%5 D=ETH\&commit=OK. (Accessed 21 May 2019).

16. Central Statistical Agency (CSA) and ORC Macro Inc. Ethiopia Demographic and Health Survey 2016. Addis Ababa, Ethiopia and Calverton: USACSA and ORC Macro; 2016

17. Dara Malo District Health Office. Annual public health emergency management report of Dara Malo District. Wacha Town: Dara Malo District Health Office; 2019.

18. Bloom DE, Cadarette D. Infectious disease threats in the 21st century: strengthening the global response. Front Immunol. 2019;10:549. https://doi. org/10.3389/fimmu.2019.00549.

19. Alamaw SD, Kassa AW, Gelaw YA. Pertussis outbreak investigation of Mekdela district, south Wollo zone, Amhara region, north-West Ethiopia. BMC Res Notes. 2017;10(1):420. 
20. Tesfa F. Outbreak investigation, pertussis outbreak investigation of Abergelle district, wag Himra zone, Amhara region, north - East Ethiopia in compiled body of works in field epidemiology: Addis Ababa university (unpublished thesis for master of public health in field epidemiology); 2018.

21. World Health Organization. How to gather and use information from the community. Global Programme for Vaccines and Immunization in Cameroon. Geneva: World Health Organization; 2001.

22. Daniel W. Biostatistics: A Foundation for analysis in the health sciences. 9th ed. New York: Wiley; 2009.

23. Regassa N, Bird Y, Moraros J. Preference in the use of full childhood immunizations in Ethiopia: the role of maternal health services. Patient preference and adherence. 2019;13:91.

24. Damtew ZA, Karim AM, Chekagn CT, Zemichael NF, Yihun B, Willey BA, Betemariam W. Correlates of the Women's development Army strategy implementation strength with household reproductive, maternal, newborn and child healthcare practices: a cross-sectional study in four regions of Ethiopia. BMC Pregnancy Childbirth. 2018;18(Suppl 1):373.

25. Turner AG, Magnani RJ, Shuaib M. A not quite as quick but much cleaner alternative to the expanded Programme on immunization (EPI) cluster survey design. Int J Epidemiol. 1996;25:198-203.

26. Kansas Department of Environment \& Health. Pertussis (whooping cough) investigation quideline. Topeka: KDHE; 2012

27. SPSS, Inc. IBM SPSS statistics base 20. Chicago: SPSS Inc.; 2011

28. Federal Ministry of Health $(\mathrm{FMOH})$. Health Data Quality Training Module: Facilitator Manual. Addis Ababa: Federal Ministry of Health; 2018.

29. Guimarães LM, da Costa Carneiro EL, Carvalho-Costa FA. Increasing incidence of pertussis in Brazil: a retrospective study using surveillance data. BMC Infect Dis. 2015;15(1):442.

30. Kilgore PE, Salim AM, Zervos MJ, Schmitt HJ. Pertussis: microbiology, disease, treatment, and prevention. Clin Microbiol Rev. 2016;29(3):449-86.

31. Wendelboe AM, Van Rie A, Salmaso S, Englund JA. Duration of immunity against pertussis after natural infection or vaccination. Pediatr Infect Dis J. 2005;24(5):S58-61.

32. Thisyakorn U, Tantawichien T, Thisyakorn C, Buchy P. Pertussis in the Association of Southeast Asian Nations: epidemiology and challenges. Int J Infect Dis. 2019;87:75-83.

33. Zerbo O, Bartlett J, Goddard K, Fireman B, Lewis E, Klein NP, et al. Pediatrics. 2019:144(1)

34. Datta SS, Toikilik S, Ropa B, Chidlow G, Lagani W. Pertussis outbreak in Papua New Guinea: the challenges of response in a remote geotopographical setting. Western Pacific surveillance response J. 2012;3(4):3.

35. Soofie N, Nunes MC, Kgagudi P, van Niekerk N, Makgobo T, Agosti Y, Hwinya C, Pathirana J, Madhi SA. The burden of pertussis hospitalization in HIV-exposed and HIV-unexposed south African infants. Clin Infect Dis. 2016; 63(suppl 4):S165-s173.

36. Barger-Kamate B, Deloria Knoll M, Kagucia EW, Prosperi C, Baggett HC, Brooks WA, Feikin DR, Hammitt LL, Howie SR, Levine OS, et al. Pertussisassociated pneumonia in infants and children from low- and middleincome countries participating in the PERCH study. Clin Infect Dis. 2016; 63(suppl 4):S187-s196.

37. Drug Administration and Control Authority of Ethiopia (DACAE). Standard treatment guideline for health centers. Addis Ababa: DACAE; 2010.

38. Theodoridou M, Hadjipanagis A, Persianis N, Makri S, Hadjichristodoulou C. Pertussis outbreak detected by active surveillance in Cyprus in 2003. Eurosurveillance. 2007;12(5):11-2.

39. Dellepiane N, Pagliusi S. Registration experts working group. Challenges for the registration of vaccines in emerging countries: differences in dossier requirements, application and evaluation processes. Vaccine. 2018;36(24): 3389-96.

40. Craig AT, Joshua CA, Sio AR, Lauri M, Kaldor J, Rosewell AE, Schierhout G. Towards effective outbreak detection: a qualitative study to identify factors affecting nurses' early warning surveillance practice in Solomon Islands. BMC Health Serv Res. 2018;18(1):702.

41. Endriyas M, Solomon T, Belayhun B, Mekonnen E. Poor quality data challenges conclusion and decision making: timely analysis of measles confirmed and suspected cases line list in southern nations nationalities and People's region, Ethiopia. BMC Infect Dis. 2018;18(1):77.

42. Patriarca PA, Biellik RJ, Sanden GA, Burstyn DG, Mitchell PD, Silverman PR, Davis JP, Manclark CR. Sensitivity and specificity of clinical case definitions for pertussis. Am J Public Health. 1988;78(7):833-6.
43. Guiso N, Taieb F. Pertussis in low and medium income countries: a pragmatic approach. In: Fedele G, Ausiello C, editors. Pertussis infection and vaccines, Advances in experimental medicine and biology, springer, Cham, vol. 1183; 2019. p. 137-49.

\section{Publisher's Note}

Springer Nature remains neutral with regard to jurisdictional claims in published maps and institutional affiliations.
Ready to submit your research? Choose BMC and benefit from:

- fast, convenient online submission

- thorough peer review by experienced researchers in your field

- rapid publication on acceptance

- support for research data, including large and complex data types

- gold Open Access which fosters wider collaboration and increased citations

- maximum visibility for your research: over $100 \mathrm{M}$ website views per year

At BMC, research is always in progress.

Learn more biomedcentral.com/submissions 